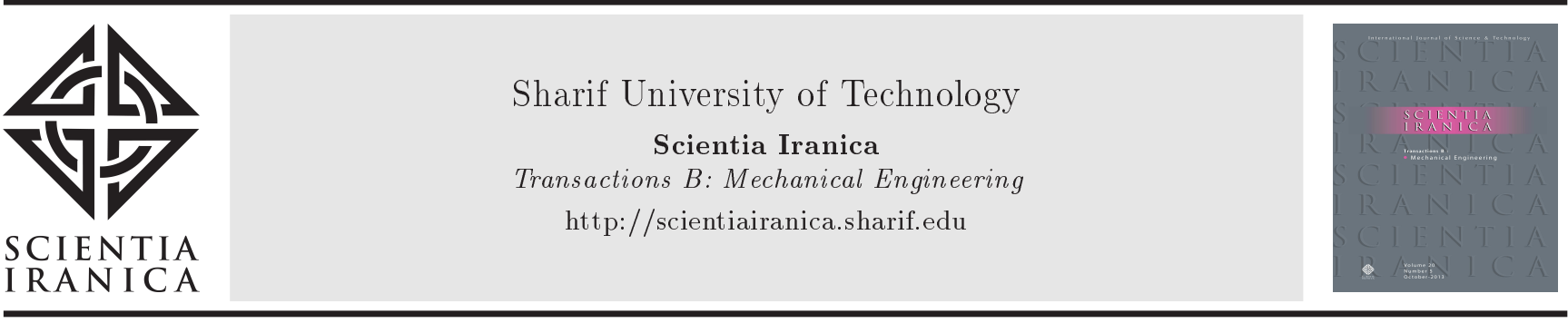

\title{
Particle rotation effects in Cosserat-Maxwell boundary layer flow with non-Fourier heat transfer using a new novel approach
}

\author{
M.B. Hafeez ${ }^{\mathrm{a}, *}$, M.S. Khan ${ }^{\mathrm{a}}$, I.H. Qureshi ${ }^{\mathrm{a}}$, J. Alebraheem ${ }^{\mathrm{b}}$, \\ and A. Elmoasry ${ }^{\mathrm{b}, \mathrm{c}}$ \\ a. Department of Applied Mathematics and Statistics, Institute of Space Technology, Islamabad, 44000, Pakistan. \\ b. Department of Mathematics, College of Sciences Al Zufli, Majmaah University, Majmaah, 11952, Saudi Arabia. \\ c. Department of Mathematics, Faculty of Sciences, Aswan University, 81528, Aswan, Egypt.
}

Received 7 November 2018; received in revised form 7 February 2020; accepted 13 July 2020

\author{
KEYWORDS \\ Boundary layer flow; \\ Non-Fourier heat \\ transfer; \\ Buoyancy effects; \\ Particle rotations; \\ Cosserat Maxwell \\ fluid; \\ Galerkin-Petrov finite \\ element method.
}

\begin{abstract}
The present study employs a non-classical approach to investigate different physical effects of Cosserat-Maxwell fluid flow on the mechanism of non-Fourier heat transfer mechanism. Furthermore, a new numerical approach was used and outlined to compute and analyze the behavior of such kinds of flow. In particular, continuous GalerkinPetrov discretization scheme was included in this study using the shooting method to obtain the numerical algorithm and solve the stagnation point flow of Cosserat-Maxwell fluid with Cattaneo-Christov heat transfer. The mathematical description of the physical problem was presented in the form of Partial Differential Equations (PDEs) governing the flow mechanism. Further, suitable transformations were utilized to describe the governing PDEs in the forms of their respective ordinary differential equations. Numerical experiments were performed for a specific case with weak concentrations of the flow near the stretching surface, thereby allowing the microelement to rotate and generate vortex flow near the stretching surface. Buoyancy effects along with other interesting physical effects were calculated, and the numerical results were presented for various fluidic situations. Several significant case studies were carried out to ensure the validity of the obtained results. Moreover, the results were validated against those available in the limiting classical continuum case in the literature and good agreement was found between them.

(C) 2021 Sharif University of Technology. All rights reserved.
\end{abstract}

\section{Introduction}

Several constitutive models for the rheology of nonNewtonian fluids have been proposed so far. Maxwell

\footnotetext{
*. Corresponding author.

E-mail addresses: bilalhafeez44@yahoo.com (M.B. Hafeez); m.sabeel@iba-suk.edu.pk (M.S.Khan); imran_haider707@yahoo.com (I.H. Qureshi); j.alebraheem@mu.edu.sa (J. Alebraheem); a.elmoasry@mu.edu.sa (A. Elmoasry)
}

fluid model functions based on relaxation theory, whereas the micropolar fluid model exhibits translational and rotational effects under spin gradient and vortex viscosities. The present study aims to combine both types of constitutive relationships and analyze the simultaneous impacts of viscoelasticity (memory effects), spin gradient, and vortex viscosity on translational and rotational motions in the presence of thermal changes. A variety of engineering applications of Maxwell fluid flow and heat transfer analysis in the literature have been already discussed [1-3] through which different interesting phenomena have been de- 
scribed. Such fluid flow has considerably been the focus of attention due to their practical significance [4,5], especially in in manufacturing industry [6], cooling systems [7], thermal efficient systems [8], automobile industry [9], chemical engineering [10], and powder technology. In all these considerations [1-10] as well as the studies in the Refs. [11,12], the physical models were considered in the absence of independent microrotational motions of the fluid particles. However, the micro-rotational motions of the fluid particles play a significant role in the dynamics of the flow and gain significance while modeling the flow mechanism and getting a true picture of the boundary layer flows in the present context. In 1909, Cosserat brothers introduced the theory of micropolar continuum, [13] which, for the first time, considered the independent micro-rotational motions of the particles and discussed the kinetic and kinematic relations and the associated balance laws in detail. Their original contribution was documented in French and reviewed late in (1912).

Das [14], Ibrahim and Zemedu [15], Ashraf and Batool [16], Triphathy et al. [17], and Yasin et al. [18] were among several researchers who took into account the independent micro-rotational motion of the particles while analyzing the boundary layer flows in their studies. In these investigations, the main focus was put only on the heat conduction, and the Fourier approach proposed by Lian-Cun et al. was taken into consideration [19]. The studies and references mentioned in [14-19] pointed to only the classical Fourier's law of heat conduction, but it could not predict the thermal relaxation phenomenon. Having this deficiency in mind, Cattaneo [20] and Christov [21] suggested that the modified Fourier's law of heat conduction could be employed to examine the thermal relaxation phenomenon in thermal transport of fluid regimes. However, this novel model has not been employed much so far. Some studies have examined the applications of the modified Fourier's law of heat conduction; however, these models did not incorporate the independent motions. Although few studies employed the Cattaneo-Christov heat transfer model, such models did not incorporate the independent microrotational motions of the particles and analyzing them was limited to the case of classical continuum. For instance, Mahapatra et al. [22] analyzed the impact of free stream velocity on the transportation of momentum in Magnetohydrodynamics (MHD) flow of power law fluid towards the stretching surface. To theoretically study the stagnation point fluid flow of an Upper-Convected Maxwell (UCM), Sadeghy et al. [23] employed the Chebyshev pseudo-spectral collocation point method to discuss the flow behavior under elasticity number. UCM model was also investigated to determine the mixed convection in the presence of magnetic field by Kumari and Nath [24]. Han et al.
[25] employed the homotopy analysis method to suggest the approximate analytical solution to the coupled flow problem in the UCM fluid with heat transfer through the non-Fourier heat transfer approach. They measured the effects of elasticity number, slip parameter, Prandtl number, and thermal relaxation time on the hydrodynamic and thermal boundary layers and compared their results with those of the Fourier heat flux model. Recently, Han et al. [25] studied the boundary layer flow of Maxwell fluids from a stretching sheet using the Cattaneo-Christov heat flux model. Sheikholeslami et al. [26] described the effects of thermal radiation on the transport of nanofluid in the presence of magnetic field. Ismael and Ghalib [27] considered the characteristics of natural convection of double diffusive flow in a cavity. Ghaffarpasand [28] discussed the Soret and Dufour effects on the flow of double convection in a lid-driven cavity. Ghaffarpasand [29] investigated the effects of MHD of the flow of non-Newtonian liquid in the heated cavity. Alinia et al. [30] studied the convection phenomenon of waterbased nanofluid on the inclined square. Sheikhzadeh et al. [31] considered the transport mechanism of mass and heat of Newtonian liquid in a cavity. Amini et al. [32] conducted an analysis of heat and mass in a cavity filled with liquid, considering the magnetic field. Arefmanesh et al. [33] discussed the phenomenon of thermal enhancement in nanofluid residing under the square cavity. The dynamics of electrically conducting fluid in the presence of magnetic field is totally different from that of electrically non-conducting fluid. Several researchers have investigated the phenomenon of the shear fluids dynamics. For example, Kaneez et al. [34] discussed the slip effects on the MHD flow of micropolar fluid using a mixture of hybrid nanoparticles. Fatunmbi and Okoya [35] studied the viscoelasticity under the influence of applied magnetic field with variable thermal conductivity and non-uniform heat source. Srinivasacharya and Mendu [36] analyzed the free convective flow of micropolar fluid and the corresponding thermal radiation in the presence of the magnetic field. Raftari and Yildirim [37] examined the flow through porous medium, employed the homotopy perturbation method to compute the UCM fluid flow with different physical effects, and obtained a semianalytical solution in the form of infinite power series.

The present study aimed to extend work of Mushtaq et al. [38] to the case of non-classical continuum and described the fluid flow by means of the Cosserat continuum theory. To this end, an additional independent degree of freedom was incorporated into the existing model, thereby providing information about the rotational microstructure of the fluidic medium under study. Moreover, a new numerical approach based on the continuous Galerkin-Petrov discretization method embedded with shooting method was introduced in 
detail, and an algorithmic description was provided to evaluate the solutions of such boundary layer flow problems. The effects of Cosserat on the skin-friction coefficient, Nusselt number, hydrodynamic boundary layer, thermal boundary layer, and micro-rotational boundary layer were also elaborated. The obtained results were validated through a comparison between these results and those in the limiting case in the literature. This study is organized as follows: In Section 2, the mathematical description of the physical model in the form of flow governing equations is given. In Section 3, the new numerical method cGPshooting is presented and the algorithm is used for numerical implementation. In Section 4, the findings are presented in the form of figures and tables. Finally, Section 5 concludes the study based on the obtained results.

\section{Problem formulation}

Consider a two-dimensional viscoelastic (Maxwell) and laminar fluid flow within the framework of non-classical continuums. The flow is adjacent to a vertical sheet flattened along the $x$-axis and normal to the $y$-axis. The flow velocities along the $x$ and $y$ axes are $u$ and $v$. Assume that the vertical sheet is stretched with a velocity of $u_{w}(x)=a x$, whereas the flow velocity of the fluid is $u_{e}(x)=c x$ with $a$ and $c$ as positive constants. Further, let $T_{\infty}$ be the temperature of the resting fluid and $T_{w}(x)=T_{\infty}+b x$ the wall temperature with $b$ as a positive constant. A transverse magnetic field of strength $B_{0}$ is exposed to the conducting viscoelastic fluid. Based on the above-mentioned assumptions and the Oberback-Boussinesq approximations, the Maxwell flow governing equations are described as follows:

$$
\begin{gathered}
\frac{\partial u}{\partial x}+\frac{\partial v}{\partial y}=0 \\
u \frac{\partial u}{\partial x}+v \frac{\partial u}{\partial y}+\lambda_{1}\left[u^{2} \frac{\partial^{2} u}{\partial x^{2}}+v^{2} \frac{\partial^{2} u}{\partial y^{2}}+2 u v \frac{\partial^{2} u}{\partial x \partial y}\right] \\
=u_{e} \frac{d u_{e}}{d x}+\left(v+\frac{k}{\rho}\right) \frac{\partial^{2} u}{\partial y^{2}}-\frac{\sigma}{\rho} B_{0}{ }^{2} \\
\quad\left(u-u_{e}+\lambda_{1} \frac{\partial v}{\partial y}\right)+\frac{k}{\rho} \frac{\partial N}{\partial y} \pm g B_{T} \\
\quad\left[\left(T-T_{\infty}\right)+\lambda_{1}\left\{u \frac{\partial T}{\partial x}+v \frac{\partial T}{\partial y}-\frac{\partial u}{\partial x}\left(T-T_{\infty}\right)\right\}\right] \\
u \frac{\partial N}{\partial x}+v \frac{\partial N}{\partial y}=\frac{\gamma *}{\rho j} \frac{\partial^{2} N}{\partial y^{2}}-\frac{k}{\rho j}\left(2 N+\frac{\partial u}{\partial y}\right), \\
\rho c_{p}\left(u \frac{\partial T}{\partial x}+v \frac{\partial T}{\partial y}\right)=-\nabla \cdot q,
\end{gathered}
$$

where $\lambda_{1}$ represents the fluid relaxation time, $\rho$ the fluid density, $v$ the kinematics viscosity, $B_{T}$ thermal expansion coefficient, $\gamma^{*}$ the viscosity of spin gradient, $j$ the micro inertial per unit mass, $k$ the vortex viscosity, and $c_{p}$ the specific constant of heat capacity. Although the positive sign in Eq. (2) is related to the situation where the thermal buoyance effect is assisting the flow, i.e., the thermal buoyancy force is in the upward direction of the fluid flow, the negative sign stands for the opposing flow situation where the thermal buoyance force is opposite to the flow direction. In Eq. (4), the heat flux $q$ is calculated through the thermal relaxation time $\lambda_{2}$ effect and CattaneoChristov heat flux equation given below:

$$
q+\lambda_{2}\left(q_{t}+v \cdot \nabla q-q \cdot \nabla v+(\nabla \cdot v)\right)=-\kappa \nabla T .
$$

Based on the Fourier law of heat conduction [19] and Eq. (5), it is possible to solve [22] a single equation at a temperature using the mechanism of heat transfer. Thus, Eq. (4) takes the form of:

$$
\begin{aligned}
u \frac{\partial T}{\partial x} & +v \frac{\partial T}{\partial y}+\lambda_{2}\left\{\left(u \frac{\partial u}{\partial x}+v \frac{\partial u}{\partial y}\right) \frac{\partial T}{\partial x}\right. \\
& +\left(u \frac{\partial v}{\partial x}+\frac{\partial v}{\partial y}\right) \frac{\partial T}{\partial y}+u^{2} \frac{\partial^{2} T}{\partial x^{2}}+v^{2} \frac{\partial^{2} T}{\partial y^{2}} \\
& \left.+2 u v \frac{\partial^{2} T}{\partial x \partial y}\right\}=\alpha \frac{\partial^{2} T}{\partial y^{2}} .
\end{aligned}
$$

In addition, the flow of the problem at hand is governed by the set of Eqs. (1)-(3), and Eq. (6) along with the following boundary conditions:

$$
\begin{aligned}
& u(x, 0)=u_{w}(x), \quad v(x, 0)=0, \quad T(x, 0)=T_{w}, \quad \text { and } \\
& N=-N_{0} \frac{\partial u}{\partial y} \quad \text { as } \quad y \rightarrow 0 \quad u(x, y) \rightarrow u_{e}(x), \\
& T(x, y) \rightarrow T_{\infty} \quad \text { and } \quad N \rightarrow 0 \quad \text { as } \quad y \rightarrow \infty .
\end{aligned}
$$

Following the transformations, we have:

$$
\begin{aligned}
& \psi=\left(v x u_{e}\right)^{1 / 2} f(\xi), \quad \theta(\xi)=\frac{T-T_{\infty}}{T_{w}-T_{\infty}}, \\
& \xi=\left(\frac{c}{v}\right)^{1 / 2} y, \quad \text { and } \quad N=\sqrt{\frac{c^{3}}{v}} x g(\xi),
\end{aligned}
$$

where $\psi(x, y)$ is the stream function which makes it feasible to calculate the stream velocities $u=\frac{\partial \psi}{\partial y}$ and $v=-\frac{\partial \psi}{\partial x}$. Further, using Eqs. (1)-(3), Eq. (6), and Eq. (7), we arrive at the following set of the coupled nonlinear ordinary differential equations:

$$
\begin{aligned}
(1+k & \left.-\beta f^{2}\right) f^{\prime \prime \prime}+\left(1+2 \beta f^{\prime}\right) f f^{\prime \prime}-f^{2}+\frac{c^{2}}{a^{2}}-M f^{\prime} \\
& +M \frac{c}{a} M \beta f f^{\prime \prime}+k h^{\prime}+\lambda\left(\theta-\beta f \theta^{\prime}\right)=0
\end{aligned}
$$




$$
\begin{aligned}
& \left(1+\frac{k}{2}\right) g^{\prime \prime}+\left(f g^{\prime}-f^{\prime} g\right)-k\left(2 g+f^{\prime \prime}\right)=0 . \\
& \frac{1}{P r} \theta^{\prime \prime}+f \theta^{\prime}-\gamma\left(f^{2} \theta^{\prime \prime}+f f^{\prime} \theta^{\prime}\right)=0 .
\end{aligned}
$$

Along with the associated boundary conditions:

$$
\begin{aligned}
& f(0)=0, \quad f^{\prime}(0)=1, \quad \theta(0)=1, \\
& g(0)=-N_{0}, f^{\prime \prime}(0) \quad \text { at } \quad \xi=0, \quad f^{\prime \prime}(\xi) \rightarrow \lambda, \\
& g(\xi) \rightarrow 0, \quad \theta(\xi) \rightarrow 0, \quad \text { as } \quad \xi \rightarrow \infty,
\end{aligned}
$$

where $\lambda$ is called a Richardson number responsible for the mixed convection, $\mathrm{Pr}$ is the Prandtl number, $G r_{x}$ denotes the local Grashof number, $\operatorname{Re}_{x}$ is the local Reynolds number, $M$ represents the parameter of the magnetic interaction, $\beta$ is the fluid relaxation time, and $\gamma$ is the thermal relaxation time defined as:

$$
\begin{aligned}
& \lambda=\frac{\mathrm{Gr}_{x}}{\operatorname{Re}_{x}^{2}}, \quad \operatorname{Pr}=\frac{v}{\alpha}, \quad \operatorname{Gr}_{x}=\frac{g B_{T}\left(T_{w}-T_{\infty}\right) x^{3}}{v^{3}}, \\
& \operatorname{Re}_{x}=\frac{u_{w} x}{v}, \quad \beta=\lambda_{1} a, \quad \gamma=\lambda_{2} a, \quad M=\sigma B_{0}{ }^{2} / \rho a .
\end{aligned}
$$

The parameter $\lambda$ is responsible for determining the assisting and opposing flow situations. The buoyancy force acts in the direction of the free stream velocity in both halves $(x>0$ and $x<0)$ of the fluid domain where $T_{w}>T_{\infty}$ and $T_{w}<T_{\infty}$, respectively, in case $\lambda$ is positive. However, in case the values for $\lambda$ are negative, the buoyancy force acts in the opposite direction to the flow stream within both halves of fluid domain. The former condition is known as the assisting flow and the latter as the opposing flow situations. The physical quantities of interest, skin friction coefficient $C_{f}$, and Nusselt number $\mathrm{Nu}_{x}$ are defined through the following relations [4]:

$$
C_{f}=\frac{2 \tau_{w}}{\rho u_{w}^{2}}, \quad \mathrm{Nu}_{x}=\frac{x q_{w}}{\kappa\left(T_{w}-T_{\infty}\right)},
$$

where $\tau_{w}$ and $q_{\omega}$ are the wall stress and wall heat flux calculated according to the relations given below:

$$
\begin{aligned}
& \tau_{w}=\left[(\mu+k) \frac{\partial u}{\partial y}+k N\right]_{y=0}, \\
& q_{w}=-\left.\rho c_{p} \kappa \frac{\partial T}{\partial y}\right|_{y=0} .
\end{aligned}
$$

Now, by using Eq. (7) and substituting Eq. (14) into Eq. (13), the following relations are obtained:

$$
\begin{aligned}
& f^{\prime \prime}(0)=C_{f} \sqrt{\operatorname{Re}_{x}} / 2\left(1+\left(1-N_{0}\right) k\right), \\
& -\theta^{\prime}(0)=\frac{\mathrm{Nu}_{x}}{\sqrt{\operatorname{Re}_{x}}} .
\end{aligned}
$$

\section{Numerical procedure}

The system of nonlinear differential equations in Eqs. (9)-(11) is first transformed into a system of firstorder coupled differential equations, as shown in the following:

$$
\left\{\begin{array}{l}
f^{\prime} \\
f^{\prime \prime} \\
\frac{f f^{\prime \prime}\left(1+2 \beta f^{\prime}\right)+f^{\prime 2}-\frac{c^{2}}{a^{2}}+M f^{\prime}-M \frac{c}{a}-M \beta f f^{\prime \prime}-k g^{\prime}-\lambda\left(\theta-\beta f \theta^{\prime}\right)}{1+k-\beta f^{2}} \\
\theta^{\prime} \\
\left\{\frac{p r\left[-\gamma\left(f f^{\prime} \theta^{\prime}\right)-f \theta^{\prime}\right]}{1-p r \gamma f^{2}}\right\} \\
g^{\prime} \\
\left\{\frac{k\left(2 g+f^{\prime \prime}\right)-\left(f g^{\prime}-f^{\prime} g\right)}{1+k}\right\}
\end{array}\right.
$$

The above system of differential equations and initial/boundary conditions, as observed in Eq. (12), can be numerically treated using the continuous GalerkinPetrov finite element discretization scheme [39] embedded with the shooting method. The procedure involves choosing appropriate initial guess for the unknown values and shoot out the unknown initial values to finally establish the following convergence criterion:

$$
\frac{\sum_{k=1}^{N}\left|\varphi_{k}^{i}-\varphi_{k}^{i-1}\right|}{\left|\sum_{k=1}^{N} \varphi_{k}^{i}\right|}<\varepsilon,
$$

where $\varepsilon$ is the tolerance and is equal to $10^{-16}$.

Then, the boundary value problem under study is completely transformed into the initial value problem with known initial conditions. The method can be then implemented based on the following numerical algorithm.

Step 1: Initialize the constants and physical parameters: $\lambda, \frac{c}{a}, \operatorname{Pr}, \beta, \gamma, N_{0}$, and $M$.

Step 2: Based on the results obtained by Khan and Hackl [39], obtain the discrete form of the problem in terms of the following nonlinear coupled algebraic equations:

$$
\left\{\begin{aligned}
\mathbf{G}_{e n}^{1}= & \frac{1}{2} \mathbf{G}_{e n}^{0}+\frac{1}{2} \mathbf{G}_{e n}^{2}+\frac{h_{e n}}{8} \\
& \left\{\mathbf{F}\left(\xi_{e n, 0}, \mathbf{G}_{e n}^{0}\right)-\mathbf{F}\left(\xi_{e n, 2}, \mathbf{G}_{e n}^{2}\right)\right\} \\
\mathbf{G}_{e n}^{2}= & \mathbf{G}_{e n}^{0}+\frac{h_{e n}}{6} \\
& \left\{\mathbf{F}\left(\xi_{e n, 0}, \mathbf{G}_{e n}^{0}\right)+4 \mathbf{F}\left(\xi_{e n, 1}, \mathbf{G}_{e n}^{1}\right)\right. \\
& \left.+\mathbf{F}\left(\xi_{e n, 2}, \mathbf{G}_{e n}^{2}\right)\right\}
\end{aligned}\right.
$$

where $\mathbf{G}$ is the unknown vector of the state variables defined as $\mathbf{G}=\left\{f, f, f^{\prime \prime \prime}, \theta, \theta^{\prime}, g, g^{\prime}\right\}$.

Step 3: Take $\mathbf{F}$ from Step 2 and calculate the 
unknown conditions $f^{\prime \prime}(0), \theta^{\prime}(0)$, and $g^{\prime}(0)$ through consideration of the boundary conditions in Eq. (12) as well as the shooting method.

Step 4: Set the vector $\mathbf{G}^{0}=\left\{0,1, f^{\prime \prime}(0), 1, \theta^{\prime}(0)\right.$, $\left.-N_{0} f^{\prime \prime}(0), g^{\prime}(0)\right\}$.

Step 5: Discretize the domain into $N$ number of elements by choosing a step size $\Delta \xi$.

Step 6: Set the initial condition $\mathbf{G}_{e n}^{0}=\mathbf{G}^{0}$.

Step 7: Initialize the element number $e n=1$ and run loop over the number of elements.

Step 8: Determine the $\mathbf{G}_{e n}^{1}$ from the first set of discretized equations, as described in Step 3, in terms of $\mathbf{G}_{e n}^{2}$.

Step 9: Calculate the vector $\mathbf{G}_{e n}^{2}$ using the second set of algebraic equations in Step 3 and Step 9.

Step 10: Solve the resulting system of nonlinear algebraic equations for the numerical values of $\mathbf{G}_{e n}^{2}$ using Newton's Raphson iteration method. Here, double precision, i.e., $10^{-16}$, is taken into account in calculating the numerical values.

Step 11: Back-substitute the obtained numerical values of $\mathbf{G}_{e n}^{2}$ from Step 11 in the symbolic expression of $\mathbf{G}_{e n}^{1}$. from Step 9 to calculate the numerical values of $\mathbf{G}_{e n}^{1}$.
Step 12: Now, evaluate the solution at Element Number (EN) using the obtained numerical values of $\mathbf{G}_{e n}^{1}$ and $\mathbf{G}_{e n}^{2}$.

Step 13: Update $\xi_{n}$ by $\xi_{\text {en }} \leftarrow \xi_{\text {en }}+\Delta \xi$, i.e., the element number $e n=e n+1$.

Step 14: Update the initial solution for the next elemental calculation by $\mathbf{G}_{e n}^{0}=\mathbf{G}_{e n-1}^{2}$.

Step 15: If $e n \leq N$, go to Step 9 .

Step 16: Stop when $e n \leq N$, i.e., the maximum number of elements is reached.

\section{Results and discussion}

In this section, the results of the newly proposed approach in the previous section are presented and discussed for different fluidic situations using the extended physical model. To validate the numerical results from the new method, a comparison was made between them and already available ones in the literature in the limiting cases as shown in Tables 1 and 2 and good agreement was found. In Table 1, the numerical values for the skin-friction obtained by the present approach in both cases of classical and Cosserat continuum are compared with the observations of Mahapatra et al. [22], Mushtaq et al. [38], Abel et al. [40], Megahed [41], and Mustafa et al. [42]. Since Mahapatra et al. [22], Mushtaq et al. [38], Abel et al. [40], Megahed [41], and Mustafa et al. [42] considered only the Cauchy continuum case, the present results associated with

Table 1. Comparison of $\left|f^{\prime \prime}(0)\right|$ when $\beta=\lambda=0$ and $c / a=0.2$.

\begin{tabular}{ccccc}
\hline \multirow{2}{*}{$\boldsymbol{M}$} & $\begin{array}{c}\text { Mahapatra } \\
\text { et al. [22] }\end{array}$ & $\begin{array}{c}\text { Mushtaq } \\
\text { et al. [38] }\end{array}$ & \multicolumn{2}{c}{ Present } \\
\hline 0.0 & 0.9181 & 0.91811 & 0.91791350588175 & 0.741856888183653 \\
0.5 & 1.0768 & 1.0768 & 1.06986810515671 & 0.842793107493588 \\
1.0 & 1.2156 & 1.21562 & 1.21767143866621 & 0.939191158766544 \\
1.5 & 1.3404 & 1.34038 & 1.34152725658221 & 1.03084000097355 \\
2.0 & 1.4546 & 1.45460 & 1.45515584635630 & 1.11772592957313 \\
3.0 & 1.6569 & 1.65979 & 1.65996619373285 & 1.27870365608736 \\
5.0 & 2.0085 & 2.00847 & 2.00849192001708 & 1.55648999030354 \\
10 & 2.6894 & 2.68944 & 2.68943613615677 & 2.10365134600581 \\
20 & 3.6922 & 3.69223 & 3.69222943304935 & 2.91401293540116 \\
40 & 5.1412 & 5.14123 & 5.14123390107202 & 4.08963211219135 \\
60 & 6.2635 & 6.26356 & 6.26356067033267 & 5.00219893291212 \\
80 & 7.2136 & 7.21333 & 7.21333104760744 & 5.73902130930504 \\
100 & 8.052 & 8.05184 & 8.05183930090621 & 6.54195437262581 \\
200 & 11.3491 & 11.35042 & 11.3505855387580 & 10.0278439430753 \\
\hline
\end{tabular}


Table 2. Comparison of $\left|f^{\prime \prime}(0)\right|$ for different values of $\beta$ when $\lambda=c / a=0$.

\begin{tabular}{ccccccc}
\hline \multirow{2}{*}{$\boldsymbol{\beta}$} & $\begin{array}{c}\text { Abel } \\
\text { et al. [40] }\end{array}$ & Megahed & Mustafa & Mushtaq & \multicolumn{2}{c}{ Present } \\
\cline { 6 - 7 } & {$[\mathbf{4 1}]$} & et al. [42] & et al. [38] & Classical & Cosserat \\
\hline 0.0 & 0.999962 & 0.999978 & 1.000000 & 1.000000 & 1.00076082956 & 0.954146985398 \\
0.4 & 1.051948 & 1.051945 & 1.051890 & 1.051921 & 1.05806011921 & 1.035599065705 \\
0.6 & 1.1501850 & 1.101848 & 1.101903 & 1.101789 & 1.09935448030 & 1.073365939394 \\
0.8 & 1.196692 & 1.150160 & 1.150137 & 1.150168 & 1.14968674191 & 1.111079164489 \\
1.2 & 1.285257 & 1.285253 & 1.283563 & 1.285324 & 1.28492852852 & 1.222569142847 \\
1.6 & 1.368641 & 1.368641 & 1.368758 & 1.368715 & 1.36910768291 & 1.294653449232 \\
2.0 & 1.447617 & 1.447616 & 1.447651 & 1.447639 & 1.44695810765 & 1.364520479170 \\
\hline
\end{tabular}

Table 3. Numerical values of the Nusselt number and skin-friction coefficient for the assisting and opposing flow situations with $\operatorname{Pr}=7$ and $N=0.5$ in the classical continuum.

\begin{tabular}{|c|c|c|c|c|c|c|c|c|}
\hline \multirow{2}{*}{$\boldsymbol{K}$} & \multirow{2}{*}{$c / a$} & \multirow{2}{*}{$\beta$} & \multirow{2}{*}{$\lambda$} & \multirow{2}{*}{$M$} & \multicolumn{2}{|c|}{$\left|f^{\prime \prime}(0)\right|$} & \multicolumn{2}{|c|}{$-\theta^{\prime}(0)$} \\
\hline & & & & & $\lambda=-0.5$ & $\lambda=0.5$ & $\lambda=-0.5$ & $\lambda=0.5$ \\
\hline 0 & 0.3 & 0 & 0.2 & 2 & 1.40725270813239 & 1.20977015711368 & 2.16931498152699 & 2.20672702968185 \\
\hline- & - & 1 & - & - & 1.74264236950249 & 1.44372528129071 & 2.04558502360165 & 2.11599424450654 \\
\hline- & - & 3 & - & - & 2.30930535228941 & 1.85741169196654 & 1.87001400674594 & 1.98662535911318 \\
\hline- & - & - & 0.3 & - & 2.30016901555095 & 1.86593831713946 & 2.01795650403543 & 2.12468485815556 \\
\hline- & - & - & 0.5 & - & 2.29280752514308 & 1.87557752089149 & 2.22704155923115 & 2.36381808122583 \\
\hline- & - & - & 0.7 & - & 2.28779469909713 & 1.88165992307584 & 2.36727302778284 & 2.51455749264917 \\
\hline- & - & - & - & 2 & 2.30930574903686 & 1.85741225199383 & 1.87001724296058 & 1.98662833908239 \\
\hline- & - & - & - & 4 & 2.77044488973785 & 2.37308420968131 & 1.79156974131263 & 1.88536035298158 \\
\hline- & - & - & - & 6 & 3.16169285200454 & 2.80192338946728 & 1.73834703766939 & 1.81669193888226 \\
\hline
\end{tabular}

Cauchy/classical continuum strongly agree with their observations. Added to this, the values for the skinfriction are also presented for different magnetic field strengths in the case of Cosserat continuum. In Table 2, the values for the skin friction are computed by changing the fluid relaxation time using the presented method for classical and Cosserat continuum flow cases. Of note, the numerical values for skin friction are in good agreement with those obtained by Mahapatra et al. [22], Mushtaq et al. [38], Abel et al. [40], Megahed [41], and Mustafa et al. [42]. The numerical values of the Nusselt number and skin friction coefficient in the mechanisms of both assisting and opposing flows, with the consideration of classical continuum model, are shown in Table 3 . In this study, $\lambda=0.5$ and $\lambda=-0.5$ were attributed to the assisting and opposing flows, respectively, along with the other varying parametric values mentioned in Table 3. The values for the skin-friction coefficient in the classical-Newtonian model are smaller than those in the classical-Maxwell model for both assisting and opposing flow situations. However, the Nusselt number in the classical-Newtonian is higher than that in the classical-Maxwell flow situation for both assenting and opposing flows. Furthermore, an increase in the fluid relaxation time can increase the skin friction coefficient and decrease the Nusselt number in both assisting and opposing flows. Moreover, the skin friction coefficient and Nusselt number showed different and similar trends, respectively, with a variation in the thermal relaxation time of the classical-Maxwell model in both assisting and opposing flow situations. The skin friction coefficient and the Nusselt number also showed increasing and decreasing trends with a variation in the magnetic field strength for both flow cases, respectively. In Table 4, the computational values of the Nusselt number and skin friction coefficient are presented for the case of Cosserat-Newtonian and Cosserat-Maxwell models with both assisting and opposing flow cases. As observed, the skin friction coefficient and Nusselt number followed increasing and decreasing trends, respectively, in both the flow cases of the CosseratMaxwell model. An increase in the parameter $c / a$, i.e., the slow straining motion near the stretching sheet surface, causes a decrease in the skin friction coefficient and an increase in the Nusselt number in both flow cases. Furthermore, in case the free stream velocity of the fluid is larger than the stretching sheet velocity, 
Table 4. Numerical values of the Nusselt number and skin friction coefficient for the assisting and opposing flow situations with $\operatorname{Pr}=7$ and $N=0.5$ in the case of the Cosserat continuum.

\begin{tabular}{|c|c|c|c|c|c|c|c|c|}
\hline \multirow{2}{*}{$\boldsymbol{K}$} & \multirow{2}{*}{$c / a$} & \multirow{2}{*}{$\beta$} & \multirow{2}{*}{$\lambda$} & \multirow{2}{*}{$M$} & \multicolumn{2}{|c|}{$\left|f^{\prime \prime}(0)\right|$} & \multicolumn{2}{|c|}{$-\theta^{\prime}(0)$} \\
\hline & & & & & $\lambda=-0.5$ & $\lambda=0.5$ & $\lambda=-0.5$ & $\lambda=0.5$ \\
\hline 1 & 0.5 & 2 & - & 5 & 1.33541197002137 & 1.18837185522573 & 2.19238172825388 & 2.19220505464772 \\
\hline- & - & 3 & - & - & 1.51315102799921 & 1.29709539921589 & 2.14805830008455 & 2.1774813153426 \\
\hline- & - & 5 & - & - & 1.82719931086475 & 1.54626996631386 & 2.08432107353733 & 2.13791766783263 \\
\hline- & 0.7 & - & 0.2 & - & 0.99828966382055 & 0.78151851554522 & 2.31661447322238 & 2.35748679575081 \\
\hline- & 0.9 & - & - & - & 0.42295842943167 & 0.20217317711392 & 2.48644203201436 & 2.52421843969779 \\
\hline- & 1.1 & - & - & - & 0.220276216679379 & 0.35870641912426 & 2.65393221435977 & 2.66466579357901 \\
\hline- & - & - & - & 2 & 1.19788618857614 & 0.93468958183648 & 2.20535622743233 & 2.27071454913583 \\
\hline- & - & - & - & 4 & 1.40397773473827 & 1.18006431889827 & 2.16407693189669 & 2.21319518721975 \\
\hline- & - & - & - & 6 & 1.59677400242844 & 1.38849886071684 & 2.12700162083437 & 2.17052598511705 \\
\hline 2 & 0.5 & 0 & - & 5 & 0.790189463990634 & 0.728013525754088 & 2.33886601334564 & 2.34974536146465 \\
\hline- & - & 1 & - & - & 0.924872998551556 & 0.831030442666131 & 2.29505885304854 & 2.31429459814682 \\
\hline- & - & 3 & - & - & 1.200455328729081 & 1.044478770822643 & 2.21416559495069 & 2.24762570461335 \\
\hline 3 & - & - & 0.2 & - & 1.02482527470701 & 0.903603244593128 & 2.25490095514955 & 2.28264902086647 \\
\hline 5 & - & - & - & - & 0.836072906309726 & 0.751260759563752 & 2.30346962886805 & 2.32366987055364 \\
\hline 7 & - & - & - & - & 0.735943247584222 & 0.670689523823102 & 2.33072676063138 & 2.34657309646533 \\
\hline- & - & - & - & 2 & 0.946982250600673 & 0.775308801851554 & 2.27444030439275 & 2.31487747477604 \\
\hline- & - & - & - & 4 & 1.123117503012457 & 0.960820058877354 & 2.23226348873461 & 2.26746181675246 \\
\hline- & - & - & - & 6 & 1.273687462476613 & 1.125213028300431 & 2.19743490901293 & 2.23054973249857 \\
\hline
\end{tabular}

the skin friction coefficient and Nusselt number gain smaller and larger values in the opposing and assisting flows, respectively. However, the Nusselt number is assigned a larger value when the free stream velocity is higher than the stretching sheet velocity for both the flow cases. Increasing and decreasing trends in the skin friction and Nusselt number were observed with an increase in the strength parameter of the magnetic field. Cosserat parameter $\kappa$ affects the skin friction and Nusselt number with the different values of the magnetic parameter $M$. As observed, for the same value of magnetic parameter, for the small Cosserat parameter, the values of the skin friction coefficient and Nusselt number were higher than those for large Cosserat parameter, where both had smaller values in both of the flow cases. Moreover, increasing the Cosserat parameter would decrease the skin friction and increase the Nusselt number for both flow situations. Figure 1 shows the skin-friction graphs in the classical and Cosserat fluid flow cases where the obtained curves are compared with the available results in literature for different values of the magnetic field strength and fluid relaxation time. As observed, with the new calculations using the extended model of classical continuum, the skin friction gets lower values in the case of Cosserat continuum.

In some practical applications in manufacturing

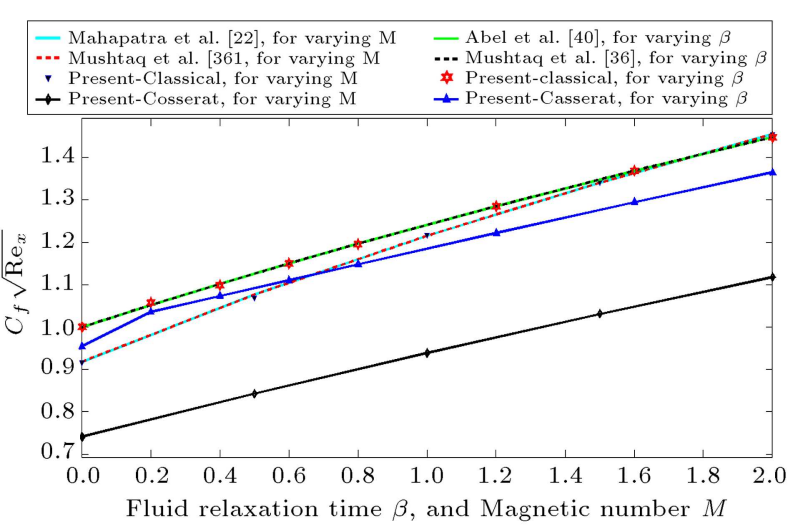

Figure 1. Comparison between the present and different models in the limiting case skin friction for different values of fluid relaxation time $\beta$ and magnetic number $M$.

processes in industry, the variation of the Prandtl number is responsible for controlling the cooling rate. In Figure 2, four different kinds of fluids are considered and temperature curves through $\theta$ are plotted for different values of the Prandtl number. For all those four cases, the width of the thermal boundary layer becomes shorter as the Prandtl number gets larger; hence, the heat transfer rate on the stretching surface increases with an increase in the Prandtl number. Moreover, the temperature profiles in the case of both 


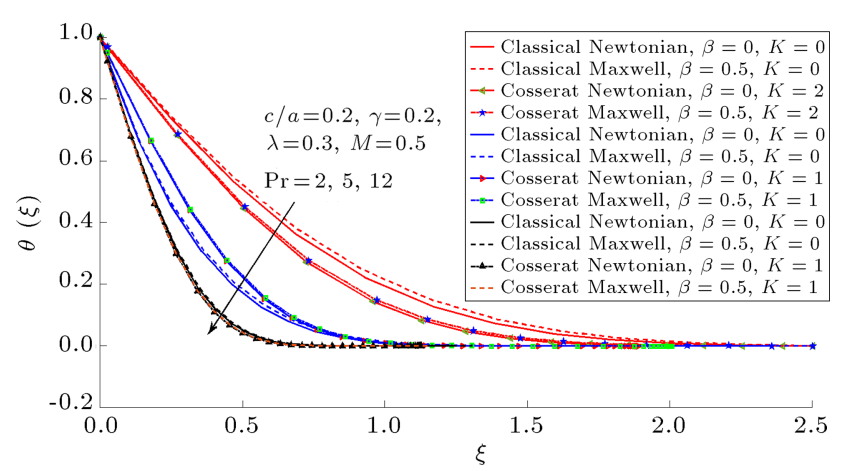

Figure 2. Comparison of classical-Newtonian, classical-Maxwell, Cosserat-Newtonian, and Cosserat-Maxwell fluids for thermal profiles with different values of Prandtl number.

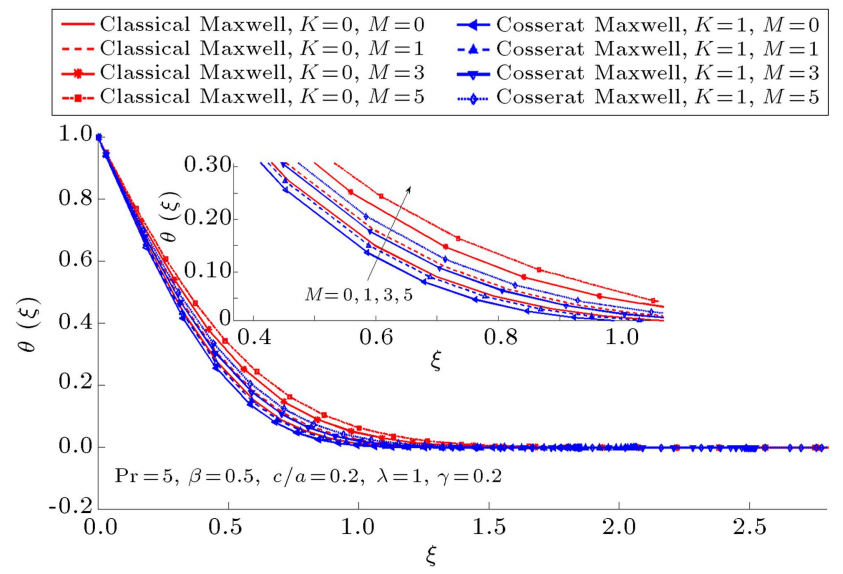

Figure 3. Comparison of thermal profiles using classical-Maxwell and Cosserat-Maxwell fluid for different values of magnetic number.

Cosserat-Newtonian and Cosserat-Maxwell fluids are always lower than those of classical-Newtonian and classical-Maxwell ones. Therefore, the heat transfer rates in the Cosserat-Newtonian and Cosserat-Maxwell fluids are higher than those in the classical-Newtonian and classical-Maxwell fluids, respectively, for the same choice of material. Figure 3 depicts the temperature profiles of thermal boundary layers for different values of the magnetic parameters; it is observed that the width of the thermal boundary layer increases with an increase in the magnetic field strength in both of the classical and Cosserat-Maxwell fluid cases. The reason behind this temperature rise in the thermal boundary layer near the stretching surface is that when the magnetic field strength increases, the velocity of the fluid flow near the stretching surface will decrease, thereby the less heated fluid will move away from the stretching surface boundary and the wall temperature gradient will be small, resulting in the rise of temperature near this surface. The velocity component represented by $f^{\prime}(\xi)$ is shown in Figure 4 for varying magnetic field strengths and fixed convection parameter $\lambda$ for the two different cases of classical and Cosserat-Maxwell fluid flows. Two different flow situations are considered. In the first situation, when $c / a=0.2<1$, the free stream velocity is smaller than the stretching speed of the surface, thus applying a drag force to the fluid. In the second situation, $c / a=1.5>1$ is chosen. In this case, the free stream velocity of fluid is greater than that on the stretching surface; thus, the fluids on the surface are dragged onto the stretching surface. In Figure 4, the velocity profiles are determined and shown for both of the flow situations. In the first case, when $c / a=0.2$, the hydrodynamic boundary layer decreases with increasing the magnetic parameter for both of the classical and Cosserat-Maxwell fluids; however, in the latter case, the hydrodynamic boundary layer increases with an increment in the magnetic parameter for both of the classical and Cosserat-Maxwell fluids. Moreover, it is observed that the velocity profiles in the case of classical-Maxwell fluids are significantly greater than the velocity profiles in the case of Cosserat-Maxwell fluid in both of the flow situations, i.e., $c / a<1$ and $c / a>1$. The flow of classical-Maxwell and CosseratMaxwell fluids in case of the assisting flow situation is considered and the difference between the two is analyzed, as shown in Figure 5 where different values of $c / a$ are taken into consideration. It is observed that the thickness of the velocity boundary layer in case of Cosserat-Maxwell fluid is always smaller than that of classical-Maxwell fluid at the same value of $c / a$. The micro rotational velocity curves in the case of assisting flow for varying values of magnetic parameters are shown in Figure 6 for Cosserat-Newtonian and Maxwell fluids. It is observed that the micro rotational velocity layer increases upon increasing the magnetic parameter close to the stretching surface in case of both the Cosserat-Newtonian and Cosserat-Maxwell fluids. Moreover, the micro rotational motions of the fluid particles are more dominant in the case of Maxwell fluid near the stretching surface than the simple CosseratNewtonian fluid. Thus, the fluid relaxation time affects the microrotations motion of the fluid particles significantly. The micro-rotational velocity profile in case where the free stream velocity of the fluid is greater than the velocity of the stretching surface is plotted in Figure 7. Two different cases, i.e., the CosseratNewtonian and Cosserat-Maxwell, are considered with different magnetic parameters. It is observed that the micro rotational motion of the fluid particle in both of the Cosserat-Maxwell and Cosserat-Newtonian fluids is getting faster upon increase in the magnetic field strength. Interestingly, compared to the pervious case (see Figure 6) where the speed of the stretching surface is greater than the free stream velocity, the fluid particles undergo the opposite orientation of the motion. Moreover, this counter rotational observation of the fluid particle is derived from the change of the 


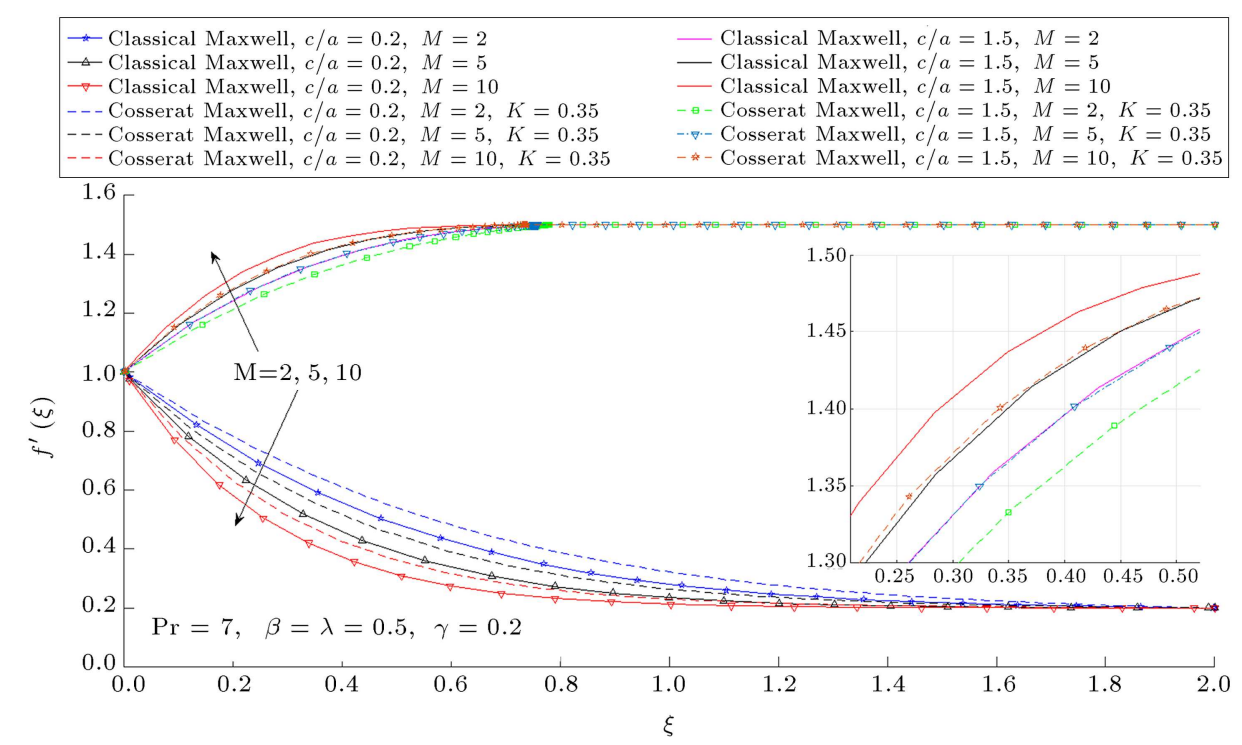

Figure 4. Comparison of velocity profiles using classical-Maxwell and Cosserat-Maxwell fluids for varying values of magnetic field strength and for different values of $c / a$.

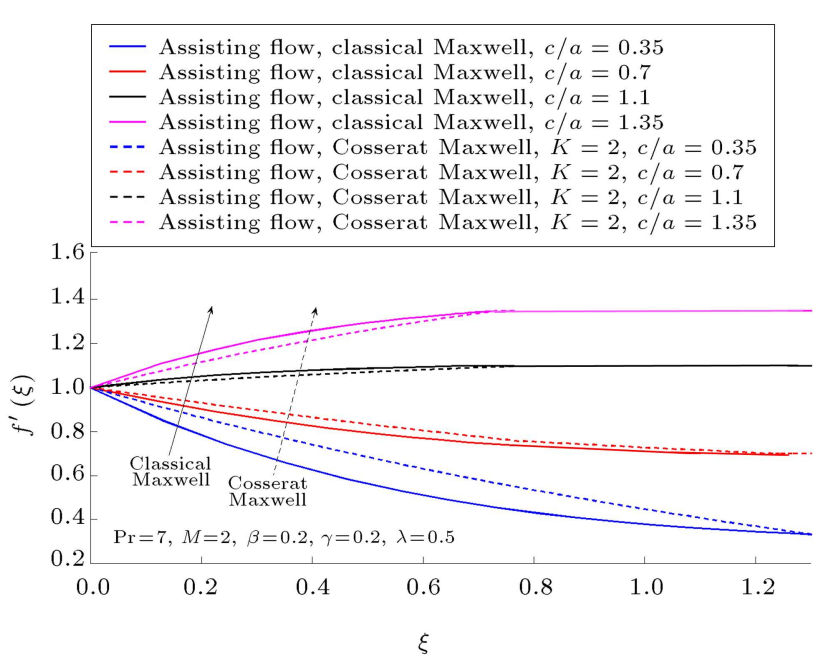

Figure 5. Comparison of velocity profiles using classical-Maxwell and Cosserat-Maxwell in the assisting flow with varying values of $c / a$.

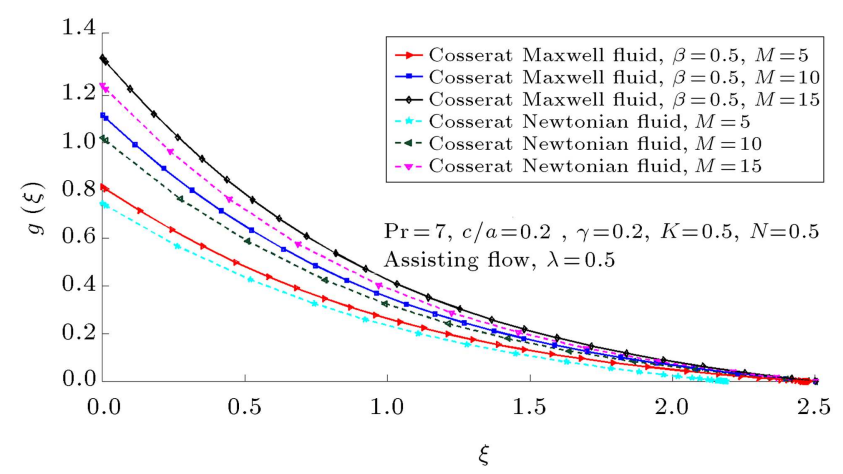

Figure 6. Microrotational velocity profile for varying magnetic numbers in the case when the free stream velocity is lower than the speed of the stretching surface.

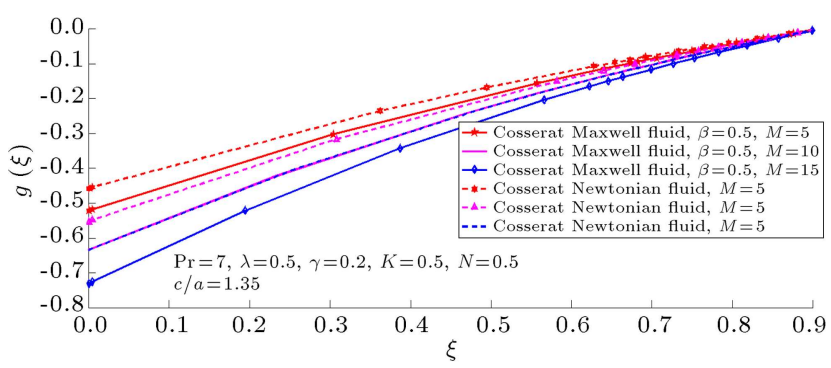

Figure 7. Microrotational velocity profile for varying magnetic numbers in the case when the speed of the stretching surface is lower than the free stream velocity.

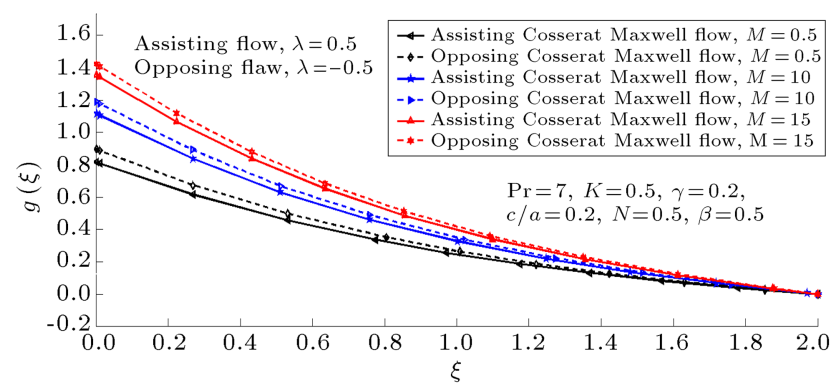

Figure 8. Microrotational velocity profiles with different magnetic field strengths in the cases of the assisting and opposing flow situations.

drag provided by different velocities of the free stream and the stretching surface. Moreover, the fluid particles get larger rotations in the case of Cosserat-Maxwell fluid than those in the case of the Cosserat-Newtonian fluid for the same material parameter.

In Figure 8, two different situations (i.e., assisting and opposing) are considered for the Cosserat-Maxwell fluid flow under the variation of magnetic field strength 


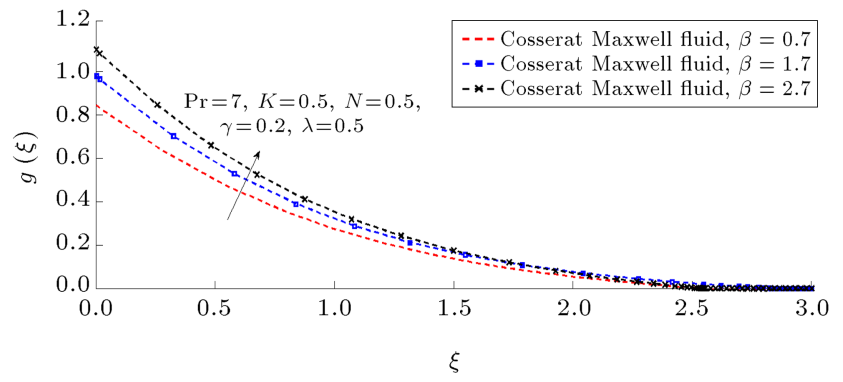

Figure 9. Microrotational velocity profile for varying values of the fluid relaxation time.

in the case where the free stream velocity is greater than the speed of the stretching surface. The micro rotational velocity boundary layer is shown for both of the flow situations given that the fluid particles get larger micro-rotations in the case of the assisting flow than the opposing flow for the same magnetic parameter. Moreover, by increasing the magnetic field strength, the micro-rotation motion of the particles becomes larger for both situations in the CosseratMaxwell fluid flow.

The micro-rotation velocity profiles of CosseratMaxwell fluid are shown in Figure 9, where the effect of fluid relaxation time is shown in the case of the assisting flow. As observed earlier, incrementing the fluid relaxation time allows fluid particles to gather greater energy to enhance the micro rotational momentum of the Cosserat-Maxwell fluid, thereby increasing the micro rotational motions.

\section{Conclusion remarks}

This study employed the viscoelastic and micropolar rheological models to solve the governing problem caused by the application of non-Fourier Law of heat conduction. The shooting method was used for numerical simulations. The simulation could read the following key observations:

- Thermal and momentum relaxation characteristics could be useful while restoring the fluid deformation and thermal changes by which the fluid and thermal equilibria could be restored;

- The spinning motion caused a significant decrease in translation motion and, then, diffusion of wall momentum into fluid slowed down. However, the rotation of solid structures showed an ascending tendency with an increase in the vortex viscosity parameter;

- A significant reduction in the thickness of thermal boundary layer against an increase in the value of Prandtl number was observed;

- As observed, the rate of heat transfer in CosseratNewtonian and Cosserat-Maxwell fluids was higher than that in classical-Newtonian and classicalMaxwell fluids;

- The micro-rotational motions of the fluid particles were more dominant in Cosserat-Maxwell fluid near the stretching surface than the simple Newtonian fluid for the same material parameters, indicating a significant effect of fluid relaxation time on the micro-motions of the fluid particles;

- Fluid particles had larger micro-rotations in the assisting flow than the opposing flow for the same values of magnetic field strength. Moreover, increasing the magnetic field strength would increase the micro-rotational motions of particles in both of the flow situations in Cosserat-Maxwell fluid flow.

\section{Acknowledgment}

The authors, except M.S. Khan, extend their appreciation to the Deanship of Scientific Research at Majmaah University for funding this work under project number (RGP 20194).

\section{Nomenclature}

a Stretching rate

$b \quad$ Temperature constant

$B_{0} \quad$ Magnitude of magnetic induction

$B_{T} \quad$ Thermal expansion coefficient

c Stretching rate

$c_{p} \quad$ Specific heat

$C_{f} \quad$ Skin friction

$g \quad$ Gravitational acceleration

$G r_{x} \quad$ Grashof number

j Micro inertial

$k \quad$ Vortex viscosity

$M \quad$ Hartmann number

$N \quad$ Micro rotation

$N u_{X} \quad$ Nusselt number

$\mathrm{Pr} \quad$ Prandtl number

$q \quad$ Heat flux

$q_{w} \quad$ Wall heat flux

$R e_{x} \quad$ Local Reynolds number

$T \quad$ Temperature

$T_{w} \quad$ Wall temperature

$T_{\infty} \quad$ Ambient temperature

$u_{e} \quad$ Fluid velocity

$u_{w} \quad$ Wall velocity

$(u, v, w) \quad$ Velocity component in $x, y$ and $z$ direction

$(x, y, z) \quad$ Space coordinates 
Fluid relaxation time

$\gamma$

Thermal relaxation time

Spin gradient viscosity

Electrical conductivity

Fluid relaxation time

Thermal relaxation time

\section{References}

1. Eringen, A.C. "Theory of micropolar fluids", J. Math. Mech., 16, pp. 1-18 (1966).

2. Uddin, Z., Kumar, M., and Harmand, S. "Influence of thermal radiation and heat generation absorption on MHD heat transfer flow of a micropolarfluid past a wedge with hall and ion slip currents", Thermal Sci., 18, p. S489 (2014).

3. Mukhopadhyay, S. and Bhattacharyya, K. "Unsteady flow of a Maxwell fluid over a stretching surface in presence of chemical reaction", J.E. Math. Soc., 20(3), pp. 229-234 (2012).

4. Abel, M.S., Tawade, J.V., and Nandeppanavar, M.M. "MHD flow and heat transfer for the upper-convected Maxwell fluid over a stretching sheet", Meccanica, 1, pp. $25-38$ (2013).

5. Shah, S., Hussain, S., and Sagheer, M. "MHD effects and heat transfer for the UCM fluid along with Joule heating and thermal radiation using CattaneoChristov heat flux model", AIP Adv., 6, p. 085103 (2016).

6. Renardy, M. "High Weissenberg number boundary layers for the upper convected Maxwell fluid", J. NonNewtonian Fluid Mech., 68, pp. 125-133 (1997).

7. Olaru, I. "A study of the cooling systems and fluid flow simulation in metal cutting processing", IOP Conf. Ser. Mater. Sci. Eng., 227, p. 012086 (2017).

8. Abel, M.S. and Mahesha, N. "Heat transfer in MHD viscoelastic fluid flow over a stretching sheet with variable thermal conductivity non-uniform heat source and radiation", Appl. Mathematical Model., 32(10), pp. 1965-1983 (2008).

9. Guilmineau, E. "Computational study of flow around a simplified car body", J. Wind Engin. and Industrial Aerodynamics, 6(7), pp. 1207-1217 (2008).

10. Levenspiel, O. "Chemical reaction engineering", Chem. Res., 38(11), pp. 4140-4143 (1999).

11. Rana, S., Nawaz, M., and Qureshi, I.H. "Numerical study of hydrothermal characteristics in nano fluid using KKL model with Brownian motion", Sci. Iran., 26(3), pp. 1931-1943 (2019).

12. Atif, S.M., Hussain, S., and Sagheer, M. "Effect of thermal radiation on MHD micropolar Carreau nanofluid with viscous dissipation, Joule heating, and internal heating", Sci. Iran., Transaction F., Nanotechnology, 26(6), pp. 3875-3888 (2019).
13. Eringen, A.C. "Theory of thermomicro fluid", J. Math. Anal. Appl., 138, pp. 480-496 (1972).

14. Das, K. "Slip effects on MHD mixed convection stagnation point flow of a micropolar fluid towards a shrinking vertical sheet", Comput. Math. Appl., 63(1), pp. $255-267$ (2012).

15. Ibrahim, W. and Zemedu, C. "MHD nonlinear mixed convection flow of micropolar nanofluid over nonisothermal sphere", Math. Prob. Eng., 2020(1), p. 4735650 (2020).

16. Ashraf, M. and Batool, K. "MHD flow and heat transfer of a micropolar fluid over a stretchable disk", J. Theor. Appl. Mech., 51(1), pp. 25-38 (2013).

17. Tripathy, R.S., Dash, G.C., Mishra, S.R., et al. "Numerical analysis of hydromagnetic micropolar fluid along a stretching sheet embedded in porous medium with non-uniform heat source and chemical reaction", Eng. Sci. Technol. Int. J., 19, pp. 1573-1581 (2016).

18. Yasin, M.M., Ishak, A., and Pop, I. "MHD stagnationpoint flow and heat transfer with effects of viscous dissipation joule heating and partial velocity slip", Sci. Rep., 5, p. 17848 (2015).

19. Lian-Cun, Z., Xin-Xin, Z., and Chun-Qing. L. "Heat transfer for power law non-Newtonian fluids", Chin. Phys. Lett., 23(12), p. 3301 (2006).

20. Cattaneo, C. "Sulla conduzione del calore", Atti Sem. Mat. Fis, Univ. Modena., 3(1) pp. 83-101 (1948).

21. Christov, C.I. "On frame indifferent formulation of the Maxwell-Cattaneo model of finite-speed heat conduction", Mech. Res. Commun., 36(4), pp. 481-486 (2009).

22. Mahapatra, T.R., Nandy, S.K., and Gupta, A.S. "Magnetohydrodynamic stagnation-point flow of a powerlaw fluid towards a stretching surface", Int. J. Non-Linear Mech., 44, pp. 124-129 (2009).

23. Sadeghy, K., Hajibeygi, H., and Taghavi, S.M. "Stagnation-point flow of upper-convected Maxwell fluids", Int. J. Non-Lin. Mech., 41(10), pp. 1242-1247 (2006).

24. Kumari, M. and Nath, G. "Steady mixed convection stagnation-point flow of upper convected Maxwell fluids with magnetic field", Int. J. Non-Lin. Mech., 44(10), pp. 1048-1055 (2009).

25. Han, S., Zheng, L., Li, C., et al. "Coupled flow and heat transfer in viscoelastic fluid with CattaneoChristov heat flux model", Appl. Math. Letters, 38, pp. 87-93 (2014).

26. Sheikholeslami, M., Ganji, D.D., Li, Z., et al. "Numerical simulation of thermal radiative heat transfer effects on $\mathrm{Fe}_{3} \mathrm{O}_{4}$-ethylene glycol nanofluid EHD flow in a porous enclosure", Sci. Iran., 26(3), pp. 1405-1414 (2019). 
27. Ismael, M.A. and Ghalib, H.S. "Double diffusive natural convection in a partially layered cavity with inner solid conductive body", Sci. Iran., B., 25(5), pp. 26432659 (2018).

28. Ghaffarpasand, O. "Unsteady double-diffusive natural convection with Soret and Dufour effects inside a twosided lid-driven skewed enclosure in the presence of applied magnetic field", Sci. Iran., B., 25(3), pp. 1215-1235 (2018).

29. Ghaffarpasand, O. "Effect of alternating magnetic field on unsteady MHD mixed convection and entropy generation of ferro fluid in a linearly heated two-sided cavity", Sci. Iran., 24(3), pp. 1108-1125 (2017).

30. Alinia, M., Gorji-Bandpy, M., Ganji, D.D., et al. "Two-phase natural convection of $\mathrm{SiO}_{2}$-water nano fluid in an inclined square enclosure", Sci. Iran., 21(5), pp. 1643-1654 (2014).

31. Sheikhzadeh, G.A., Heydari, R., Hajialigol, N., et al. "Heat and mass transfer by natural convection around a hot body in a rectangular cavity", Sci. Iran., 20(5), pp. 1474-1484 (2013).

32. Amini, Y., Emdad, H., and Farid, M. "Fluid-structure interaction analysis of a piezoelectric flexible plate in a cavity filled with fluid", Sci. Iran., 23(2), pp. 559-565 (2016).

33. Arefmanesh, A., Mahmoodi, M., and Nikfar, M. "Effect of position of a square-shaped heat source on buoyancy-driven heat transfer in a square cavity filled with nano fluid", Sci. Iran., 21(3), pp. 1129-1142 (2014).

34. Kaneez, H., Alebraheem, J., Elmoasry, A., et al. "Numerical investigation on transport of momenta and energy in micropolar fluid suspended with dusty, mono and hybrid nano-structures", AIP Adva., 10(4), p. 045120 (2020).

35. Fatunmbi, E.O. and Okoya, S.S. "Heat transfer in boundary layer magneto-micropolar fluids with temperature-dependent material properties over a stretching sheet", Adv. Mater. Sci. Eng., p. 5734979 (2020).

36. Srinivasacharya, D. and Mendu, D.S. "Free convection in MHD micropolar fluid with radiation and chemical reaction effects", Chem. Ind. Chem. Eng. Q., 20(2), pp. 183-195 (2014).

37. Raftari, B. and Yildirim, A. "The application of homotopy perturbation method for MHD flows of UCM fluids above porous stretching sheets", Comput. Math. Appl., 59(10), pp. 3328-3337 (2010).

38. Mushtaq, A., Mustafa, M., Hayat, T., et al. "Buoyancy effects in stagnation-point flow of Maxwell fluid utilizing non-Fourier heat flux approach", Plos One, 13(7), p. e0200325 (2018).
39. Khan, M.S. and Khan, M.I. "A novel numerical algorithm based on Galerkin-Petrov time-discretization method for solving chaotic nonlinear dynamical systems nonlinear dynamics", Nonlinear Dyn., 91(3), pp. $1555-1569$ (2018).

40. Abel, M.S., Tawade, J.V., and Nandeppanavar, M.M. "MHD flow and heat transfer for the upper convected Maxwell fluid over a stretching sheet", Mecc., 47, pp. 385-393 (2012).

41. Megahed, A.M. "Variable fluid properties and variable heat flux effects on the flow and heat transfer in a nonNewtonian Maxwell fluid over an unsteady stretching sheet with slip velocity", Chin. Phys. B., 22, p. 094701 (2012).

42. Mustafa, M., Hayat, T., and Alsaedi, A. "Rotating flow of Maxwell fluid with variable thermal conductivity an application to non-Fourier heat flux theory", Int. J. Heat Mass Transf., 106, pp. 142-148 (2017).

\section{Biographies}

Muhammad Bilal Hafeez is an MSc student of Applied Mathematics \& Statistics, Institute of Space Technology, Islamabad, Pakistan. He received his BSc (Hons) degree in Mathematics, in 2015, from International Islamic University (IIUI) Islamabad, Pakistan. His area of specialization is Fluid Mechanics. In his researches, he focuses on the enhancement of heat transfer in fluid flow. In the MSc research work, he has written three research papers. Good at computer programming, he used MATLAB and MAPLE as computational tools in his studies.

Muhammad Sabeel Khan is working as an Assistant Professor at the Department of Mathematics, Sukkur Institute of Business Administration (IBA), Pakistan. He received his $\mathrm{PhD}$ degree in Applied Mathematics (in 2013) from Ruhr University Bochum, Germany. He earned his Master's degree in Computation Mechanics and Applied Mathematics in 2009. Before this, he earned BS degree from COMSATS Institute of Information Technology Islamabad, Pakistan. His area of specialization is computational mechanics and applied mathematics and, till now, he has published many research article papers in international journals of very good repute.

Imran Haider Qureshi received his $\mathrm{PhD}$ degree from Institute of Space Technology, Islamabad, Pakistan. He received Master of Philosophy (MPhil) in Mathematics (in 2011) from Quaid-e-Azam University (QAU), Islamabad, Pakistan. He also earned the degree of Master of Science (MSc) in Mathematics from University of Gujrat, Gujrat, Pakistan. His area of interest is Computational Fluid Dynamics (CFD). He has published several research articles in 
international journals of very good repute. Using Finite Element Method (FEM), he is engaged in investigating thermo-physical properties of the Newtonian and nonNewtonian fluids.

Jawdat Alebraheem is working as an Assistant Professor at the Department of Mathematics, College of Science Al Zufli, Majmaah University, Saudi Arabia. He received his PhD, in Computational Mechanics and Applied Mathematics, in 2013 from Unversiti Sains Malaysia, Malaysia and his MS degree in Applied Mathematics from Unversiti Sains Malaysia, Malaysia, in 2009. Earlier, he received his BSc degree from Yarmouk University, Jordan. His area of specialization is mathematical modeling and until now, he has pub- lished some research papers in some top international journals.

Ahmed Elmoasry is working as an Assistant Professor in Mathematics at College of Sciences Majmaah University Zufli, Saudi Arabia. He obtained a PhD degree in Mathematics (in 2010) from South Valley University and Jacob's University Bremen, Germany. He earned his Master's degree in Pure Mathematics in 2003 from South Valley University, Egypt. Before this, he earned BSc degree from South Valley University, Aswan, Egypt. His area of specialization is Computational Fluid Dynamics (CFD) and, till now, he has published research articles in international journals of very good repute. 